PUPT-1689

hep-th/9703201

March 1997

\title{
A Note on the Transverse Five-Brane in M(atrix) Theory
}

\author{
Gilad Lifschytz \\ Department of Physics, Joseph Henry Laboratories, \\ Princeton University, \\ Princeton, NJ 08544, USA. \\ e-mail:Gilad@puhep1.princeton.edu
}

\begin{abstract}
We describe a way to compute scattering amplitudes in M(atrix) quantum mechanics, that involve the transverse five-brane. We then compute certain scattering processes and show that they have the expected $S O(5)$ invariance, give the correct transverse-five-brane mass, and agree with the supergravity result.
\end{abstract}




\section{1 introduction}

Recently there has been a proposal that M-theory [1, 2] in the infinite momentum frame can be described as the large $N$ limit of $S U(N)$ Yang-Mills quantum mechanics 3]. In subsequent work the brane [4, 5, 6] content of type IIA theory have been discussed in this frame work [7, 8, 9, 10, 11, 12]. The branes are represented as classical backgrounds. For the transverse five-brane or the type IIA $N S$-five-brane, one does not have such a representation. In this note we will describe how to compute scattering amplitudes involving transverse five-brane (we will often call it $5^{N S}$-brane), and by that confirming its existance.

Starting with the $0+1$ theory (quantum mechanics), one can T-dualise to other $d+1$ dimensional theory and then use some specific duality of that field theory, T-dual back and get some new information in the $0+1$ dimensional theory. Here we will employ this strategy in order to compute some properties of the transverse five-brane. One does not have a description of the transverse five-brane in $0+1$ dimensional theory, however as noticed in [8] there is an indirect way of describing it. Start with the configuration of the $(2+0)$-brane (sometimes called the membrane of matrix theory) in $0+1$-dimensions, and have extra three dimension be compact (not necessarily small) call them $X_{1}, X_{2}, X_{3}$. Now If we start with the $(2+0)$ in the direction $(4,5)$ and T-dualise along $X_{1}, X_{2}, X_{3}$, then we end up with a $(5+3)$-brane in $3+1$ dimensions. Now one can use the S-duality fl of $(3+1)$ Yang-Mills, which is equivalent to the S-duality of type IIB string to get to the configuration $\left(5_{N S}+3\right)$-brane, then T-dual back to get the configuration $\left(5_{N S}+0\right)$-brane in the $0+1$ dimensional theory stretching in directions $(1-5)$. Unfortunately we do not know how the S-duality acts on the commutator $\left[X_{4}, X_{5}\right]=i c$, so one does not have the description of the transverse five-brane.

Let us instead focus on some scattering amplitudes involving the transverse five-brane, as the procedure above takes us from $0+1$ theory to another $0+1$ theory (albeit with possible different parameters) one can map a scattering amplitude involving the transverse five-brane to a known amplitude, computable in $(0+1)$-dimensional matrix theory. In this fashion one can get certain information on the transverse five- brane in the context that we want. In order to do so all we need is to map known configurations in one theory to the other via the transformation $T^{3} S T^{3}$, and also to know how the parameters (coupling and size of the space) change. Of course one needs to know the exact result in the original theory in order to be able to do this as typically the coupling will change, luckily we expect the long distance scattering to be exactly computable in the matrix theory.

We start in section (2) with a review of T-duality in the context of ma-

\footnotetext{
${ }^{1}$ Branes in the IKKT [13] model were discussed in 14, 15

${ }^{2}$ For a discussion of the validity of S-duality in the limit $N \rightarrow \infty$ see [16]
} 
trix theory, then in section (3) we describe how various configurations are transformed under the $T^{3} S T^{3}$ transformation. We calculate in section (4) scattering of zero-branes from the transverse five-brane $\left(5^{N S}+0\right)$, and show that it has an $S O(5)$ invariance and agrees with the super-gravity result.From these calculation we extract the transverse-five-brane mass. We also give the results of the scattering of two Five-branes and a five-brane and an anti-fivebrane.

\section{Review of T Duality}

In this section we review the description of compactification in matrix theory on a $d$ dimensional torus $y^{3}$. We start with the $(0+1)$ dimensional field theory, If one of the nine direction is compact (with length $L$ ) one has in the theory new sectors of states, namely open strings that wind around the compact directions [22]. If instead of describing those states this way one just Fourier transform to another description, then one sees that the same theory is naturally described as a $(1+1)$ dimensional theory with the extra dimension being compactified on a circle of length $=\frac{l_{s}^{2}}{L}\left(l_{s}=\left(\frac{l_{p}}{R_{11}}\right)^{1 / 2} l_{p}\right.$, where $R_{11}$ is the radius of the eleventh direction and $l_{p}$ is the Planck length in eleven dimensions.) Given that one has $d$ dimensions compact (on a torus) one ends with a $d+1$ dimensional super Yang-Mills compactified on the dual d-torus 22]. Now this procedure is just the T-duality of type IIA (at strong coupling), as replacing winding modes with momenta modes (Fourier transform) is Tduality. In fact in the Lagrangian given a field $X_{i}$, if we Fourier transform (T-dualise) in that direction then [8]

$$
X_{i} \rightarrow i \partial_{x_{1}}+A_{i}\left(x_{i}\right)=D_{i}
$$

where $x_{i}$ is now a parameter ranging on the dual circle and $A_{i}\left(x_{i}\right)$ captures the degrees of freedom in the $i$ direction of the original brane (position, momentum).

Let us see how the T-duality acts on the branes of the theory. Starting in $(0+1)$ dimensions the basic objects can be thought as zero-branes, while in $(d+1)$ dimensions the basic objects are branes of dimension $\mathrm{d}$, as one expects. Starting with a membrane configuration $\left[X_{1}, X_{2}\right]=i c$ in $(0+1)$ dimension, if one T-dualise in directions other that $(1,2)$ then we just end with a configuration $\left[X_{1}, X_{2}\right]=i c$ in a higher dimensional super Yang-Mills theory. This configuration can easily be seen to describe a $(d+2)$-brane (of course bound to many d-branes), as one expects from T-duality. If however we Fourier transform on coordinate $X_{1}$ then one ends in a $(1+1)$ dimensional filed theory with a configuration

$$
\left[i \partial_{x_{1}}+A_{i}\left(x_{i}\right), X_{2}\right]=i c \rightarrow D_{x_{1}} X_{2}=i c .
$$

\footnotetext{
${ }^{3}$ Discussion of compactifications on other manifolds can be found in $17,18,19,20,21$
} 
Now as $x_{1}$ is the world sheet coordinate along the 1-brane (which is the elementary object of this theory) this configuration describes a 1-brane at an angle with respect to the $x_{1}$ direction. If we just T-dualise the configuration of a membrane bounded to zero-brane in Type IIA string theory then we will get a 1-brane with an angle, just like we have. What we mean when we say a string at an angle is the following. As we are on a compact space, a string configuration that is wrapped $\mathrm{n}$ times in one direction and $\mathrm{m}$ times in another direction is a string at an angle $\sim m / n$ for $n \gg m$, in the non compact space.

T-dualising now in the $X_{2}$ direction one ends up with $\left[D_{1}, D_{2}\right]=i F_{12}=i c$ a magnetic field on the world volume of a $2+1$ theory [ 8$]$. As is well known this represents zero-branes bounded to the two-branes [23], which is the Tdual of the configuration we started with.

Let us now start with a configuration of a moving zero-brane in the $0+1$ dimensional theory. This is described by a background $X_{1}=v t$ which can be recast in the form $\left[D_{t}, X_{1}\right]=i v$, Fourier transform along $X_{1}$ to get a configuration in $1+1$ dimensions of the form $\left[D_{t}, D_{1}\right]=i F_{01}=i v$ which implies the existence of a constant electric field in the $1+1$ dimensional theory as one expects from T-duality.

Start with the configuration of a bound state of a four-brane bounded to two-branes and zero-brane in the theory in $0+1$ dimensions. This is described by $\left[X_{1}, X_{2}\right]=i c_{1}, \quad\left[X_{4}, X_{3}\right]=i c_{2}$ where $c_{1}=\frac{2 \pi R_{1} R_{2}}{n_{1}} c_{2}=\frac{2 \pi R_{3} R_{4}}{n_{2}}$, and the number of zero-brane is $N=n_{1} n_{2}$. The number of membrane in the $(1,2)$ direction is $\frac{1}{i 2 \pi R_{1} R_{2}} \operatorname{Tr}\left[X_{1}, X_{2}\right]=N c_{1}=n_{2}$ and the number of membranes in the $(3,4)$ direction is $n_{1}$. Now T-dualise along $X_{1}$ one gets a configuration in $1+1$, which is $X_{2}=c_{1} x_{1}, \quad\left[X_{3}, X_{4}\right]=i c_{2}$. This describes in the matrix theory $n_{1} 3$-branes bounded to $\mathrm{N}$ 1-strings in the $x_{1}$ direction and one string at an angle $\sim c_{1}$, exactly what one expects from the string theory.

\section{The Action of $T^{3} S T^{3}$}

In this section we will describe how configuration change under the $T^{3} S T^{3}$ transformation and how the parameters of the theory change.

\subsection{Mapping of Configurations}

In order to make things clear let us always call the three directions we $\mathrm{T}$ dualise in $X_{1}, X_{2}, X_{3}$, any different direction will be labeled as $X_{i}$. As was explained before under the transformation $T^{3} S T^{3}$ a $(0+1)$ dimensional field theory is transformed back to a $(0+1)$ dimensional field theory.

Under this transformation a membrane, described by the classical background, $\left[X_{i}, X_{j}\right]=i c$ is transformed to a transverse five-brane in directions $(1,2,3, i, j)$. Take a membrane $\left[X_{1}, X_{i}\right]=i c$ under $T^{3}$ one gets $D_{1} X_{i}=i c$ 
( $c=\frac{2 \pi R_{i} R_{1}}{N}$ ) which is a configuration of $N$ three-branes in directions $(1,2,3)$ and one three-brane in directions $(2,3, i)$. Then acting by $S$ one gets the same configuration and acting again by $T^{3}$ we are back in a configuration of a membrane $\left[X_{1}, X_{i}\right]=i c$. Similar transformation can be made on other configuration and we will just give the results:

- Membrane in $i, j \leftrightarrow$ transverse five-brane in directions $(1,2,3, i, j)$, or in symbols $2_{(i, j)}+0 \leftrightarrow 5_{(1,2,3, i, j)}^{N S}+0$

- $2_{(1, i)}+0 \leftrightarrow 2_{(1, i)}+0$

- zero-brane moving in $i$ direction $\left(0+v_{i}\right) \leftrightarrow$ same

- $2_{(2,3)}+0 \leftrightarrow$ zero-brane moving in direction $1\left(0+v_{1}\right)$

- $4_{(1, i, j, k)}+2_{(i, j)}+2_{(1, k)}+0 \leftrightarrow 5_{(2,3, i, j, k)}^{N S}+5_{(1,2,3, i, j)}^{N S}+2_{(1, k)}+0$

- $4_{(1,2, i, j)}+2_{(1, i)}+2_{(2, j)}+0 \leftrightarrow$ same.

- $4_{(1,2, i, j)}+2_{(1,2)}+2_{(i, j)}+0 \leftrightarrow 4_{(1,2, i, j)}+v_{3}+5_{(1,2,3, i, j)}^{N S}+0$

- $4_{(1,2,3, i)}+2_{(12)}+2_{(3, i)}+0 \leftrightarrow 1_{i}^{N S}+v_{3}+2_{(3, i)}+0$

Given the results for the potentials between these configurations given in 10, 12 and taking care to take into account the effect of the extra compact direction one can read off the potentials between configurations that one does not have access directly in the $0+1$ dimensional field theory.

Now as $T$ and $S$ duality does not change the supersymmetry of a configuration we can also see that we can get new configurations that preserve a quarter of the supersymmetry that has relative velocity between the brane configuration. For example two stationary orthogonal $(2+0)$-branes is a supersymmetric configuration if there parameter $c$ are the same. This implies that a zero-brane moving with a certain velocity parallel to a $\left(5_{N S}+0\right)$ bound state is also supersymmetric. Similarly because a configuration of $(4+2+2+0)$ and a stationary zero-brane is supersymmetric [25, 24] for a specific choice of parameter, then all the configuration on the right-hand side off the table above plus a zero-brane are supersymmetrid'

\subsection{Parameter transformation}

Let us start with a string theory at coupling $g$, the lengths for the $(1,2,3)$ directions labeled by $L_{1}, L_{2}, L_{3}$, and lengths for the other directions $X_{j}$. Under T-duality in a $k$ direction the length $L_{k}^{\prime}=\frac{l_{s}^{2}}{L_{k}}$ and the coupling $g^{\prime}=g \frac{l_{s}}{L_{k}}$.

\footnotetext{
${ }^{4}$ Some related classical supergravity solutions can be found in 27, 26, 28.
} 
Under S duality $g^{\prime}=g^{-1}$ and all lengths transform $L^{\prime}=L\left(g^{\prime}\right)^{1 / 2}$. Starting with the above parameters under $T^{3} S T^{3}$ one ends up with

$$
\begin{aligned}
g^{\prime} & =g V_{p}^{1 / 2} \\
L_{i}^{\prime} & =L_{i} V_{p}^{-1 / 2} \\
X_{i}^{\prime} & =X_{i} V_{p}^{1 / 2}
\end{aligned}
$$

Where $V_{p}=\frac{L_{1} L_{2} L_{3}}{g l_{s}^{3}}$ is the volume in Planck units of the space $L_{1}, L_{2}, L_{3}$.

Notice that we have used the S-duality transformation for the parameter from string theory, off course with the philosophy at hand this should come from the S-duality symmetry of the Yang-Mills theory. In (3+1) Yang-Mills under S-duality the three-torus stays the same, the coupling is inverted and the scalars are multiplied by $V_{p}$ (the scalars represent the transverse space). Using then the conformal invariance we scale the torus sides by $V_{p}^{1 / 2}$ which is accompanied multiplying the scalars by $V_{p}^{-1 / 2}$ which is then just the S-duality of the string theory.

Due to the $T^{3} S T^{3}$ symmetry of the theory the phase shift [29] of a configuration must be mapped to the phase shift of the mapped configuration.

$$
A=-\int d t V\left(b^{2}+v^{2} t^{2}\right)=A^{\prime}=-\int d t^{\prime} V^{\prime}\left(b^{\prime 2}+v^{2} t^{2}\right)
$$

where $b^{\prime 2}=b^{2} V_{p}$ and $t^{\prime}=t V_{p}^{1 / 2}$.

\section{Transverse five-brane scattering}

In this section we will calculate various scattering process involving the transverse five-brane, and show that we get the correct long distance result.

We will calculate here the scattering of a zero-brane (or graviton) from the transverse five-brane. There are three different direction the zero-brane can move. First its motion can be transverse to the five-brane, this is mapped to a calculation of a zero-brane scattering off a membrane with velocity in the $(6-9)$ directions. Second the zero-brane can move parallel to the five-brane. This case is actually two-case that should agree. Movement along the $(1-3)$ directions is mapped to a computation involving two relatively stationary orthogonal two-branes, and movement along the $(4,5)$ direction is mapped to a zero-brane moving parallel to a two-brane. The last two-computations although they are different should give the same answer due to the $S O(5)$ symmetry of the transverse five-brane.

Now when computing the scattering one must take into account the three compact directions $(1,2,3)$ ] .

\footnotetext{
24

${ }^{5}$ Compact brane in matrix theory were also considered in [30], and in string theory in
} 


\subsection{Zero-brane scattering off a transverse five-brane}

Starting with a zero-brane scattering off a transverse five-brane with velocity transverse to the five-brane, we calculate a zero-brane scattering off a twobrane (described by $\left[X_{4}, X_{5}\right]=i c$ ) with three orthogonal directions much smaller that the distance between the zero-brane and two-brane. We get the long range potential [10]

$$
V=-\frac{1}{2} \int \frac{d s}{s} e^{-b^{2} s} \frac{\left(c^{2}+v^{2}\right)^{2}}{2 c \sqrt{\pi}} s^{4}\left(\frac{\pi^{3 / 2}}{L_{1} L_{2} L_{3} s^{3 / 2}}\right)
$$

Where the last factor in parenthesis is from the compactified directions, $c=$ $\frac{L_{4} L_{5}}{2 \pi N}$, and $b$ is the distance between the membrane and the zero-brane.

Let us interpret this result using five-brane variables. and using equation (4). Labeling by prime, quantities after the $T^{3} S T^{3}$ transformation and using equation (3) we find

$$
c=\frac{L_{4} L_{5}}{2 \pi N}=\frac{L_{1}^{\prime} L_{2}^{\prime} L_{3}^{\prime} L_{4}^{\prime} L_{5}^{\prime}}{2 \pi N g^{\prime} l_{s}^{3}}=\frac{L_{1}^{\prime} L_{2}^{\prime} L_{3}^{\prime} L_{4}^{\prime} L_{5}^{\prime}}{(2 \pi)^{5 / 2} N g^{\prime}}
$$

Further $(2 \pi)^{3 / 2} g L_{1} L_{2} L_{3}=g^{\prime 2}(2 \pi)^{3}$.

The potential between the zero-brane and transverse five-brane is then

$$
V_{0,5}^{\prime}=-\frac{\pi\left(c^{2}+v^{2}\right)^{2}}{4 g^{\prime}(2 \pi)^{3 / 2} c} b^{\prime-2}
$$

We will now calculate the scattering of a zero-brane off the transverse fivebrane when the zero-brane has velocity along direction 1 (or directions 2 or 3 ). the corresponding configuration is two orthogonal stationary $(2+0)$-branes. The potential for this configuration was discussed in [10]. The potential is

$V=-\frac{1}{8 \sqrt{\pi}} \int \frac{d s}{s^{3 / 2}} \frac{e^{-b^{2} s}}{\sinh c_{1} s \sinh c_{2} s}\left[4+2 \cosh 2 c_{1} s+2 \cosh 2 c_{2} s-4 \cosh \left(c_{1}-c_{2}\right) s-4 \cosh \left(c_{1}+c_{2}\right) s\right]$

Where $c_{1}=\frac{L_{2} L_{3}}{2 \pi N_{1}}$ and $c_{2}=\frac{L_{4} L_{5}}{2 \pi N_{2}}$. The long range potential when taking into account one extra transverse (to both) compact direction is,

$$
V_{\text {long }}=-\int \frac{d s}{s^{3 / 2}} \frac{e^{-b^{2} s}}{8 \sqrt{\pi} c_{1} s c_{2} s}\left(c_{1}^{2}-c_{2}^{2}\right)^{2} s^{4}\left(\frac{\sqrt{\pi}}{L_{1} s^{1 / 2}}\right)=-\frac{\left(c_{1}^{2}-c_{2}^{2}\right)^{2}}{8 c_{1} L_{1} c_{2} b^{2}} .
$$

Now after the $T^{3} S T^{3}$ transformation the two-brane in the $(2,3)$ direction is mapped to one unit of momentum along direction 1, while the zero-branes that were bound to it are mapped to zero-branes. The velocity of the cluster of $N_{1}$ zero brane will then have the velocity

$$
v=\frac{2 \pi}{N_{1} L_{1}^{\prime} T_{0}^{\prime}}
$$


Where $L_{1}^{\prime}$ is the length of direction 1 after the transformation and $T_{0}^{\prime}=\frac{2 \pi}{g^{\prime} l_{s}}$ is the zero-brane mass after the transformation. Using equation (3) one finds

$$
v=\frac{L_{2} L_{3}}{2 \pi N_{1}}=c_{1}
$$

Thus the potential between a zero-brane moving along direction 1 and a transverse five-brane stretched in directions $(1-5)$

$$
V_{5,0}^{\prime}=-N_{1} \frac{\pi\left(v_{1}^{2}-c_{2}^{2}\right)^{2}}{4(2 \pi)^{3 / 2} c_{2} g^{\prime}} b^{\prime-2} .
$$

Where $c_{2}=\frac{L_{1}^{\prime} L_{2}^{\prime} L_{3}^{\prime} L_{4}^{\prime} L_{5}^{\prime}}{(2 \pi)^{5 / 2} \mathrm{Ng}^{\prime}}$

\section{2}

If the zero-brane is moving in directions $(4,5)$ then this is mapped to a zero-brane moving in directions $(4,5)$ along a bound state of $(2+0)$-branes stretched in directions $(4,5)$. So let us calculate the later configuration in the matrix theory.

In order to calculate the phase shift one has to calculate the one-loop vacuum energy of the zero-brane quantum mechanics in the presence of a background representing the membrane and the moving zero-brane. We follow the notation of [10, 12]. One needs to calculate determinants of the operator $\left(-\partial_{0}+M^{2}\right)$, where $M^{2}$ is the mass squared of the off diagonal elements of the matrices. The membrane is defined by the coordinates $X_{5}=P$ $X_{4}=Q$ and $[Q, P]=i c=i \frac{L_{4} L_{5}}{2 \pi N}$, and the zero-brane is traveling with velocity $v$ in direction 4 . Define

$$
H=P^{2}+(Q-v t)^{2}+I b^{2}
$$

Then (in Euclidean space), for the complex bosons one has six with $M^{2}=2 H$ one with $M^{2}=2 H-4 \sqrt{c^{2}-v^{2}}$ and one with $M^{2}=2 H+4 \sqrt{c^{2}-v^{2}}$ For the fermions (in Euclidean space) one finds

$$
M_{f}^{2}=H-i c \gamma_{5} \gamma_{4}-i v \gamma_{4}
$$

thus giving eight fermions with $M_{f}^{2}=H-\sqrt{c^{2}-v^{2}}$ and eight with $M_{f}^{2}=$ $H+\sqrt{c^{2}-v^{2}}$.

Evaluating the determinants we find that the potential takes the form

$$
V=-\frac{1}{4 \sqrt{\pi}} \int \frac{d s}{s^{3 / 2}} \frac{e^{-b^{2} s}}{\sinh c s}\left[6+2 \cosh 2 \sqrt{c^{2}-v^{2}} s-8 \cosh \sqrt{c^{2}-v^{2}} s\right]
$$

This gives a long range potential (after taking into account three transverse compact directions)

$$
V_{\text {long }}=-\frac{\pi\left(c^{2}-v^{2}\right)^{2}}{4 c L_{1} L_{2} L_{3}} b^{-2}
$$


We can now calculate the potential between a transverse five-brane and a cluster of $N_{1}$ zero-branes moving with velocity in the 4-th direction

$$
V_{5,0}^{\prime}=-\frac{N_{1} \pi\left(c^{2}-v^{2}\right)^{2}}{4 c g^{\prime}(2 \pi)^{3 / 2}} b^{\prime-2}
$$

We see that equations (12) and (17) (remembering $c=c_{2}$ ) agree thus confirming the $\mathrm{SO}(5)$ invariance expected from the transverse five-brane. We will shortly also compute the mass of the transverse five-brane.

\subsection{Supergravity calculation}

Let us compare this to a supergravity calculation of a scattering of a zerobrane off a transverse five brane moving in the 11 direction (i.e from the point of view of type IIA string theory, this is the $\left(5_{N S}+0\right)$ bound state). The metric for the transverse five brane in eleven-dimensions was given in [31] (we slightly changed the notation),

$$
d s^{2}=H^{1 / 3}\left[H^{-1}\left(-d \tilde{t}^{2}+d y_{1}^{2}+d y_{2}^{2}+d y_{3}^{2}+d y_{4}^{2}+d y_{5}^{2}\right)+d \tilde{y}_{11}^{2}+d x^{i} d x_{i}\right]
$$

Here $\tilde{t}=\frac{1}{\cos \theta}\left(t-\sin \theta y_{11}\right), \tilde{y}_{11}=\frac{1}{\cos \theta}\left(y_{11}-t \sin \theta\right), H=1+\cos ^{2} \theta \frac{Q}{r^{2}}$, and $Q=\frac{M_{5}}{\cos \theta}$.

computing the potential for null geodesics [32] on this metric we find

$$
V \sim-\frac{M_{5}\left(\tilde{P}_{11}^{2}+P_{\perp}^{2}\right) \cos ^{2} \theta}{\cos \theta r^{2}}
$$

Where

$$
\tilde{P}_{11}=\frac{1}{\cos \theta}\left(P_{11}-E \sin \theta\right)
$$

and $E, P_{11}, P_{1}, P_{\perp}$ are the energy, momentum in the 11 direction, momentum in direction 1 , and momentum in the perpendicular direction of the scattered zero-brane, respectively.

We are interested in the case where $P_{\perp}=0$ but $P_{1} \neq 0$. The velocity in direction 1 is then

$$
\frac{E^{2}}{P_{11}^{2}}=1+\frac{v^{2}}{1-v^{2}}=1+(\gamma v)^{2}=\cosh ^{2} \nu
$$

The potential then becomes

$$
V \sim-\frac{M_{5}(1-\sin \theta \cosh \nu)^{2}}{\cos \theta} r^{-2}
$$

To compare to the matrix calculation we should go to the limit where the membrane velocity in the 11 direction approaches 1 , and the velocity of the 
zero brane in direction 1 is small. In this case we write $\theta=\pi / 2-c^{\prime}$. The potential at this limit is

$$
V \sim-\frac{M_{5}\left(\left(c^{\prime}\right)^{2}-v^{2}\right)^{2}}{c^{\prime}} r^{-2}
$$

This is the same as equations (12) and (17) if we identify $c=c^{\prime}$.

Let us determine $c^{\prime}$. From the supergravity solution one has

$$
\begin{aligned}
\sin \theta & =\frac{P_{11}}{E}=\frac{N M_{0}}{E} \\
E^{2} & =M_{5}^{2}+N^{2} M_{0}^{2}
\end{aligned}
$$

Where $E, P_{11}$ are the energy, and the momentum in the 11 direction of the moving five-brane (which is the eleven dimensional description of the bound state of a transverse five-brane and zero-branes) and $M_{5}, M_{0}, N$ are the mass of the five-brane mass of a zero-brane and the number of zero-brane respectively. Then we find

$$
c^{\prime}=\frac{M_{5}}{N M_{0}}
$$

From this identification and the identification from the scattering in string theory $c=c^{\prime}$ we can read off the transverse five-brane mass in the matrix calculation $\left(l_{s}^{2}=2 \pi\right)$.

$$
M_{5}=\frac{L_{1}^{\prime} L_{2}^{\prime} L_{3}^{\prime} L_{4}^{\prime} L_{5}^{\prime}}{(2 \pi)^{2}\left(g^{\prime}\right)^{2}}
$$

Which is the correct transverse five-brane mass.

If we now calculate the scattering in the supergravity with $P_{\perp} \neq 0$ and $P_{1}=0$ we will get the result from the matrix model equation (7). The agreement may also be viewed as an additional evidence for S-duality.

Notice that if we wanted to check that an elementary string will acquire a Berry phase once it is transported around the $N S$ five-brane, this would correspond to the transportation of a four-brane around a two-brane in the original theory which does acquire the berry-phase [33].

\section{4}

Similar calculations can be done for the scattering of two transverse fivebranes $\left(5^{N S}+0\right)$, this is mapped to a computation of scattering two membranes $(2+0)$. Taking care of the compact directions, the resulting potential between two membranes and between a membrane and an anti-membrane are 10

$$
\begin{aligned}
V_{2,2} & =-\frac{L_{4} L_{5} v^{4}}{8 c^{2} L_{1} L_{2} L_{3}} r^{-2} \\
V_{2, \overline{2}} & =-\frac{2 L_{4} L_{5} c^{2}}{L_{1} L_{2} L_{3}} r^{-2}
\end{aligned}
$$


Using the transformation $T^{3} S T^{3}$ this gives the potential between two moving transverse five-branes in matrix theory, and the potential between a transverse five-brane and an anti transverse five-brane, to be $\left(l_{s}^{2}=2 \pi\right)$

$$
\begin{aligned}
V_{5,5}^{\prime} & =-\frac{L_{1}^{\prime} L_{2}^{\prime} L_{3}^{\prime} L_{4}^{\prime} L_{5}^{\prime}}{g^{\prime 2}(2 \pi)^{5 / 2}} \frac{v^{4}}{4 c^{2} \pi^{1 / 2}} r^{\prime-2} \\
V_{5, \overline{5}}^{\prime} & =-\frac{L_{1}^{\prime} L_{2}^{\prime} L_{3}^{\prime} L_{4}^{\prime} L_{5}^{\prime}}{g^{\prime 2}(2 \pi)^{5 / 2}} \frac{4 c^{2}}{\pi^{1 / 2}} r^{\prime-2}
\end{aligned}
$$

and $c$ is as in equation (6). Off course one can continue and calculate scattering processes with all the states described in section (3), and probably others.

In general this way of calculating scattering amplitudes is no less powerful than the way one does that for configurations which we know how to represent them as classical backgrounds. However observing the connection between parameters given in section (3.2), one sees that we are unable to describe the limit in which the five-brane are un-compactified. This maybe related to the absence of the corresponding charge in the supersymmetry algebra of zero-branes in [11].

\section{Acknowledgments}

I would like to thank S.D. Mathur and S. Ramgoolam for helpful discussions.

\section{References}

[1] E. Witten, Nucl Phys. B443 (1995) 85, hep-th/9503124.

[2] C. Hull and P.K. Townsend, Nucl. Phys. B438 (1995) 109, hepth/9410167.

[3] T. Banks, W. Fischler, S.H. Shenkar and L. Susskind, M-theory as a matrix model: A conjecture, hep-th/9610043.

[4] J. Dai, R.G. Leigh and J. Polchinski, Mod. Phys. Lett. A4 (1989) 2073.

R.G. Leigh, Mod. Phys. Lett. A4 (1989) 2767.

[5] M.B. Green, Phys. Lett. B329 (1994) 435, hep-th/9403040.

[6] J. Polchinski, Phys. Rev. Lett. 75 (1995) 4724,

[7] L. Susskind, T Duality in M(atrix) Theory and S Duality in field theory, hep-th/9611164.

[8] O. Ganor, S. Ramgoolam and W. Taylor IV, Branes, Fluxes and Duality in M(atrix)-Theory, hep-th/9611202. 
[9] O. Aharony and M. Berkooz, Membrane Dynamics in M(atrix) Theory, hep-th/9611215.

[10] G. Lifschytz and S.D. Mathur, Supersymmetry and Membrane interactions in M(atrix) theory hep-th/9612087.

[11] T. Banks, N. Seiberg and S. Shenkar, Branes from Matrices, hepth/9612157.

[12] G. Lifschytz, Four-Brane and Six-Brane Interactions in M(atrix) Theory, hep-th/9612223.

[13] N. Ishibashi, H. Kawai, Y. Kitazawa and A. Tsuchiya, A Large-N Reduced Model as Superstring, hep-th/9612115.

[14] M. Li Strings from IIB Matrices, hep-th/9612222.

[15] I. Chepelev, Y. Makeenko and K. Zarembo, Properties of D-Branes in Matrix Model of IIB Superstring, hep-th/9701151.

[16] I.I. Kogan, G.W. Semenoff and R.J. Szabo, D-brane Configurations and Nicoli Map in Supersymmetric Yang-Mills, hep-th/9612251.

[17] S. Kachru and E. Silverstein, On Gauge Bosons in the Matrix Model Approach to $M$ Theory, hep-th/9612162.

[18] M.R. Douglas, Enhanced Gauge Symmetry in M(atrix) Theory, hepth/9612126.

[19] L. Motl, Proposal on nonperturbative Superstring Interactions, hepth/9701025.

[20] N. Kim and S. Rey, M(atrix) Theory on an Orbifold and Twisted Membranes, hep-th/9701139.

[21] M.R. Douglas, H. Ooguri and S.H. Shenker, Issues in (M)atrix Model Compactification, hep-th/9702203.

[22] W. Taylor, D-brane field theory on compact spaces, hep-th/9611042.

[23] M.R. Douglas, Branes within Branes hep-th/9512077.

[24] G. Lifschytz, Probing bound states of D-branes hep-th/9610125.

[25] M. Berkooz, M.R. Douglas and R.G. Leigh, Branes intersecting at an Angle, hep-th/9606139.

[26] J.C. Breckenridge, G. Michaud and R.C. Myers, More D-brane bound states, hep-th/9611174. 
[27] A.A. Tseytlin, Composite BPS Configurations of P-Branes in Tendimensions and Eleven-dimensions, hep-th/9702163.

[28] J.C. Breckenridge, G. Michaud and R.C. Myers, New Angles on Dbranes, hep-th/9703041.

[29] C. Bachas, D-brane dynamics, Phys. Lett. B374 (1996) 37, hepth/9511043.

[30] D. Berenstein and R. Corrado, M(atrix) Theory in Various Dimensions, hep-th/9702108.

[31] J.G. Russo and A.A. Tseytlin, Waves, Boosted Branes and BPS States in $M$-Theory, hep-th/9611047.

[32] G. Lifschytz, Comparing D-branes to black-branes, hep-th/9604156.

[33] M. Berkooz and M.R. Douglas, Five-brane in M(atrix) Theory, hepth/9610236. 